\title{
Immunization status of Iranian military recruits against Bordetella pertussis infection (whooping cough)
}

\author{
Morteza Izadi ${ }^{1}$, Shahla Afsharpaiman ${ }^{1}$, Nematollah Jonaidi Jafari ${ }^{1}$, Reza Ranjbar ${ }^{2}$, Mohammad Mahdi \\ Gooya $^{3}$, Javad Robat Sarpooshi ${ }^{1}$, Ali Akbar Esfahani ${ }^{1}$, Hamid Soheylipoor ${ }^{1}$ \\ ${ }^{1}$ Health Research Center, Baqiyatallah University of Medical Sciences, Tehran, Iran \\ ${ }^{2}$ Molecular and Biology Research Center, Baqiyatallah University of Medical Sciences, Tehran, Iran \\ ${ }^{3}$ The Ministry of Health, Tehran, Iran
}

\begin{abstract}
Introduction: Military recruits are susceptible to respiratory pathogens because of increased antibiotic resistance and the lack of an effective vaccine. The goal of the current study was to determine the immunological status of the Bordetella pertussis among conscripts in Iranian military garrisons.

Methodology: The study population consisted of 424 conscripts aged 18 to 21 years who enrolled for military service. They were selected using cluster stratified sampling from all military garrisons in Tehra, Iran. To determine the seroprevalence of infection, blood specimens from all recruits were collected and stored at $-20^{\circ} \mathrm{C}$ until assayed. All serum samples were screened for immunoglobulin $\mathrm{G}$ (IgG) antibodies against Bordetella pertussis toxin (PT) and by using enzyme-linked immunosorbent assay (ELISA).

Results: The overall prevalence of $B$. pertussis seropositivity in military recruits was 60.6 . Only 55.0\% of the recruits had low awareness about the record of vaccination against $B$. pertussis during childhood. Among 424 studied individuals, 48 recruits (11.3\%) had a positive history of whooping cough; prevalence of seropositivity in these recruits was $70.0 \%$. Among these subjects, $61.7 \%$ were referred to a physician for treatment and only $39.6 \%$ of them were administered anti-pertussis therapy.

Conclusions: Our study showed that military conscripts in Tehran garrisons were not serologically immune to pertussis and also confirmed the low awareness about vaccination and medical history related to pertussis infection in this high-risk subgroup of the Iranian population. Routine acellular booster vaccination, particularly before 18 years of age, is recommended.
\end{abstract}

Key words: Bordetella pertussis; vaccination; military; infection

J Infect Dev Ctries 2011; 5(3):224-226.

(Received 20 February 2010 - Accepted 04 July 2010)

Copyright (c) 2011 Izadi et al. This is an open-access article distributed under the Creative Commons Attribution License, which permits unrestricted use, distribution, and reproduction in any medium, provided the original work is properly cited.

\section{Introduction}

Despite the implementation of national programs for immunization against Bordetella pertussis (B. pertussis) and their acceptable vaccination coverage in many countries, this infection represents an important contribution to disease in different age subgroups, particularly in developing countries $[1,2]$. According to the reports of centers for disease control and prevention [3], B. pertussis remains the most commonly reported vaccine-preventable disease, especially in children. However, it has been shown that immunity can dramatically decline during the 12 years after vaccination, causing adolescent and adult susceptibility [4]. Recently, we assessed the immunological status of $B$. pertussis in conscripts in Iranian military garrisons.

\section{Methodology}

The study population consisted of 424 conscripts aged 18 to 21 years who enrolled for military service. Participants were selected using cluster stratified sampling from all military garrisons in Tehran. This study group is an appropriate and acceptable sample of adolescent subgroups of our population. All of them were males. Baseline characteristics of conscripts were collected by a self-administered questionnaire that included demographics characteristics, history of $B$. pertussis infection and/or vaccination against $B$. pertussis, and history of previous contact with other whooping cough patients. To determine the seroprevalence of infection, blood specimens from all recruits were collected and stored at $<-20^{\circ} \mathrm{C}$ until assayed. All serum samples were screened for IgG antibodies against $B$. pertussis toxin (PT) by enzyme-linked immunosorbent assay 
(ELISA) (IBL International GmbH, Hamburg, Germany) according to the manufacturer's instructions. Positive antibody serology titers $>20$ $\mathrm{U} / \mathrm{mL}$ were taken as a sign of immunity to $B$. pertussis, while negative $(<20 \mathrm{U} / \mathrm{mL})$ serology titers were assumed to be reflective of non-immunity [2].

\section{Results}

Among a total of 424 recruits studied, 93.6\% were single, only 57 had an academic degree and most of them (74.8\%) had secondary education. The overall prevalence of Bordetella $B$. pertussis seropositivity in military recruits was $60.6 \%$. The mean level of $\operatorname{IgG}$ against $B$. pertussis toxin at entry into service garrisons was $50.28 \pm 54.21 \mathrm{U} / \mathrm{mL}$. Of a total of 424 recruits, 167 (39.4\%) showed $<20 \mathrm{U} / \mathrm{ml}$ anti-PT IgG. Interestingly, 86 recruits (20.3\%) demonstrated levels of IgG against PT of $>80 \mathrm{U} / \mathrm{ml}$, indicating recent $B$. pertussis infection prior to enrolment.

Thirty-six recruits $(8.5 \%)$ revealed records of vaccination against $B$. pertussis during childhood and $55.0 \%$ of them had low awareness about this subject. Fourteen cases confirmed previous contact with another whooping cough patient as follows: in 9 recruits, the contact occurred at age $>15$ years; in three cases the contact occurred between 5 to 10 years of age; in the other two cases the contact occurred between 10 and 15 years of age. Among 424 studied individuals, 48 recruits $(11.3 \%)$ had a positive history of whooping cough; among them
$88.0 \%$ suffered from this infection after 15 years of age and only one patient suffered from the disease before five years of age. The prevalence of seropositivity in recruits with a previous history of whooping cough was $70.0 \%$. Among these patients, $61.7 \%$ referred to a physician for treatment and only $39.6 \%$ of them were administered anti-pertussis therapy. Regarding seasonal prevalence of whooping cough, among the 48 recruits with a history of pertussis, $41.7 \%$ and $33.3 \%$ had a history of the disease in winter and autumn, respectively. Prevalence of whooping cough in spring and summer in these subjects was $18.8 \%$ and $6.2 \%$, respectively.

Regarding medical history in the groups with different IgG levels, no significant differences were found across the groups in relation to previous history of whooping cough and previous contact with $B$. pertussis patients. History of vaccination against $B$. pertussis during childhood was similarly low in all three levels of the IgG groups (Table 1). Additionally, $52.7 \%$ of cases with negative IgG levels and $66.3 \%$ of subjects who suffered from recent infection had dramatically no awareness about their childhood vaccination against $B$. pertussis.

\section{Discussion}

To our knowledge, this is the first investigation of $B$. pertussis immunization in military conscripts in Iran. Our study confirmed that more than one-third of our cohort was susceptible to $B$. pertussis infection with $<20 \mathrm{U} / \mathrm{ml} \mathrm{IgG}$ against $B$. pertussis toxin. This

Table 1. Medical and vaccination history in the groups with different IgG levels against pertussis toxin

\begin{tabular}{clll}
\hline Medical history & $\begin{array}{l}\text { IgG level<20 } \\
(n=167)\end{array}$ & $\begin{array}{l}\text { IgG level 20-80 } \\
(n=171)\end{array}$ & $\begin{array}{l}\text { IgG level>80 } \\
(n=86)\end{array}$ \\
\hline History of pertussis infection: (n, \%) & $14(8.4)$ & $25(14.6)$ & $9(10.5)$ \\
Yes & $144(86.2)$ & $135(78.9)$ & $72(83.7)$ \\
No & $9(5.4)$ & $11(6.4)$ & $5(5.8)$ \\
Unknown & $7(4.2)$ & $4(2.3)$ & $3(3.5)$ \\
\hline Previous contact with other patients: (n, \%) & $144(86.2)$ & $136(79.5)$ & $72(83.7)$ \\
Yes & $16(9.6)$ & $31(18.1)$ & $11(12.8)$ \\
No & & & 0.451 \\
Unknown & $12(7.2)$ & $16(9.4)$ & $8(9.3)$ \\
Yes & $67(40.1)$ & $67(39.2)$ & $221(24.4)$ \\
No & $88(52.7)$ & $88(51.5)$ & $57(66.3)$
\end{tabular}

Data are presented as number (percentage) 
finding shows an unsatisfactory immunization level against whooping cough among military conscripts aged 18 to 21 years in Tehran garrisons. Furthermore, measurement of serum IgG levels in these individuals indicated that $20.3 \%$ of them had a recent $B$. pertussis infection prior to enrolment. This rate was considerably higher than that found in a similar study by Aase et al. in military recruits in Norway, which showed the recent infection rate as $8.4 \%$ [2].

Despite the reported effectiveness of the triple diphtheria-tetanus-whole cell $B$. pertussis vaccine in our population in the recent decade [5, most studied patients had low awareness about their previous vaccinations against pertussis. This low awareness may be associated with the low social level of most recruits or their low education level. In a similar study by Lopreiato et al. in Bethesda, ten percent of $B$. pertussis patients did not have complete immunization records [6. It seems that providing a national $B$. pertussis education campaign has not done enough to raise awareness of this disease in the population, especially in individuals who are enrolled as military recruits. Therefore, more education campaigns are required to increase awareness and to target recruits to inform them about $B$. pertussis infection and its adverse events.

\section{References}

1. Cherry JD (2005) The epidemiology of pertussis: a comparison of the epidemiology of the disease pertussis with the epidemiology of Bordetella pertussis infection. Pediatrics 115: 1422-1427.

2. Aase A, Herstad TK, Merino S, Brandsdal KT, Berdal BP, Aleksandersen EM, Aaberge IS (2007) Opsonophagocytic activity and other serological indications of Bordetella pertussis infection in military recruits in Norway. Clinical and Vaccine Immunology 14: 855-862.

3. Gray GC, Callahan JD, Hawksworth AW, Fisher CA, Gaydos JC (1999) Respiratory diseases among U.S. military personnel: countering emerging threats. Emerging Infectious Diseases 5: 379-385.

4. Lambert HJ (1965) Epidemiology of a small pertussis outbreak in Kent County, Michigan. Public Health Rep 80: 365-369.

5. Zarei S, Jeddi-Tehrani M, Akhondi MM, Zeraati H, Kheirkhah T, Ghazanfari M, Shokri F (2007) Immunogenicity of a triple diphtheria-tetanus-whole cell pertussis vaccine in Iranian preschool children. Iranian Journal of Immunology 4: 101-109.

6. Lopreiato JO and Moriarty R (1993) Immunization status of a military dependent population. Military Medicine 158: 371-373.

\section{Corresponding author}

Shahla Afsharpaiman

Health Research Center

Baqiyatallah University of Medical Sciences

Mollah Sadra St. Vanak Square

Tehran, Iran

Tel: 989122577137

Email:safshar@razi.tums.ac.ir

Conflict of interests: No conflict of interests is declared. 